\title{
Lessons Learned from a Major Near Miss: A Case Report Including Recommendations to Improve Future Patient Safety in Rehabilitation
}

\author{
Chelsea R. Van Zytveld \\ University of Colorado Denver, Anschutz Medical Campus \\ Jennifer W. Rodriguez \\ University of Colorado Denver, Anschutz Medical Campus \\ Tamara S. Struessel \\ University of Colorado Denver, Anschutz Medical Campus, tami.struessel@ucdenver.edu
}

Follow this and additional works at: https://nsuworks.nova.edu/ijahsp

Part of the Physical Therapy Commons

This Case Study has supplementary content. View the full record on NSUWorks here:

https://nsuworks.nova.edu/ijahsp/vol14/iss3/5

\section{Recommended Citation}

Van Zytveld CR, Rodriguez JW, Struessel TS. Lessons Learned from a Major Near Miss: A Case Report Including Recommendations to Improve Future Patient Safety in Rehabilitation. The Internet Journal of Allied Health Sciences and Practice. 2016 Jan 01;14(3), Article 5.

This Case Study is brought to you for free and open access by the College of Health Care Sciences at NSUWorks. It has been accepted for inclusion in Internet Journal of Allied Health Sciences and Practice by an authorized editor of NSUWorks. For more information, please contact nsuworks@nova.edu. 


\title{
Lessons Learned from a Major Near Miss: A Case Report Including Recommendations to Improve Future Patient Safety in Rehabilitation
}

\begin{abstract}
Background: Most medical errors can be traced to system failure, but often individual providers are blamed. Few articles in the allied health literature address the topic of error or the analysis of error using a system approach. Purpose: This case report analysis illustrates how both individual and system factors contribute to error in rehabilitation settings and how identification of these factors allows development of methods to improve future patient safety. Case Description: A young male was admitted to a rehabilitation hospital with residual impairments following recent surgical resection of a benign meningioma. He was treated daily by a physical therapist intern as part of a large interdisciplinary team. On separate occasions over a three week span, the patient presented with isolated calf tightness, right-sided and central chest pain, and fever with increased heart rate. Although the intern considered possible deep vein thrombosis (DVT), each symptom was attributed to other causes by the intern, supervising physical therapist, physician assistant, and/or physician (i.e. muscle fatigue, heartburn, infection). On day 22 , the patient was diagnosed with DVT and pulmonary embolism (PE). This event represents a major near miss due to the potential deadly outcomes of a missed DVT/PE diagnosis. The intern retrospectively completed an analysis of the event to identify contributing system factors. Outcome of Analysis: Active failures and latent system conditions on multiple levels of the healthcare system were identified as contributors to the major near miss. Discussion: The contributing factors identified in this paper are not unique to this case, intern, or facility, and may be widespread across rehabilitation settings and practitioners. Team members can and should consider how system-wide factors contribute to their own clinical actions and decisions. Once factors are identified, strategies for reducing risk can be implemented; ultimately creating a culture of safety.
\end{abstract}

\section{Author Bio(s)}

- Chelsea R. VanZytveld PT, DPT, is a licensed physical therapist in the state of Colorado, practicing in Denver, Colorado.

- Jenny Rodriguez, PT, MHS, is an Associate Professor in the Doctor of Physical Therapy Program, School of Medicine, University of Colorado Anschutz Medical Campus, Aurora, Co. She is a licensed physical therapist in the state of Colorado.

- Tamara S. Struessel, PT, DPT, is an Assistant Professor in the Doctor of Physical Therapy Program, School of Medicine, University of Colorado Anschutz Medical Campus, Aurora, Co. She is a licensed physical therapist in the state of Colorado. 


\title{
IUAHSP
}

\section{The Internet Joưnal of Allied Health Sciences and Practice}

Dedicated to allied health professional practice and education

Vol. 14 No. 3 ISSN 1540-580X

\section{Lessons Learned from a Major Near Miss: A Case Report Including Recommendations to Improve Future Patient Safety in Rehabilitation}

\author{
Chelsea R. Van Zytveld, PT, DPT \\ Jennifer W. Rodriguez, PT, MHS \\ Tamara S. Struessel, PT, DPT \\ University of Colorado \\ United States
}

\begin{abstract}
Background: Most medical errors can be traced to system failure, but often individual providers are blamed. Few articles in the allied health literature address the topic of error or the analysis of error using a system approach. Purpose: This case report analysis illustrates how both individual and system factors contribute to error in rehabilitation settings and how identification of these factors allows development of methods to improve future patient safety. Case Description: A young male was admitted to a rehabilitation hospital with residual impairments following recent surgical resection of a benign meningioma. He was treated daily by a physical therapist intern as part of a large interdisciplinary team. On separate occasions over a three-week span, the patient presented with isolated calf tightness, right-sided and central chest pain, and fever with increased heart rate. Although the intern considered possible deep vein thrombosis (DVT), each symptom was attributed to other causes by the intern, supervising physical therapist, physician assistant, and/or physician (i.e. muscle fatigue, heartburn, infection). On day 22, the patient was diagnosed with DVT and pulmonary embolism (PE). This event represents a major near miss due to the potential deadly outcomes of a missed DVT/PE diagnosis. The intern retrospectively completed an analysis of the event to identify contributing system factors. Outcome of Analysis: Active failures and latent system conditions on multiple levels of the healthcare system were identified as contributors to the major near miss. Discussion: The contributing factors identified in this paper are not unique to this case, intern, or facility, and may be widespread across rehabilitation settings and practitioners. Team members can and should consider how systemwide factors contribute to their own clinical actions and decisions. Once factors are identified, strategies for reducing risk can be implemented; ultimately creating a culture of safety.
\end{abstract}

\section{BACKGROUND:}

Error is inevitable in healthcare settings because of the complexity of the environment and the human fallibility of healthcare providers. When medical error occurs, human tendency is to look for individuals to blame..$^{1-3}$ However, most errors occur because of a recurrent set of circumstances and system conditions rather than solely the error of one person.1,2,4 The Institute of Medicine's (IOM) To Err is Human attributes the majority of patient injuries to system failure. ${ }^{1}$ The IOM's Crossing the Quality Chasm, as explained by Berwick, expands this concept by presenting four levels of the American healthcare system. ${ }^{5}$ (Figure 1) Factors at any level may contribute to error at the level of the patient's experience (Level A). ${ }^{5}$ 
Figure 1. Summary of Berwick's four levels of the American healthcare system which serves as the framework for the Institute of Medicine's Crossing the Quality Chiasm ${ }^{5}$

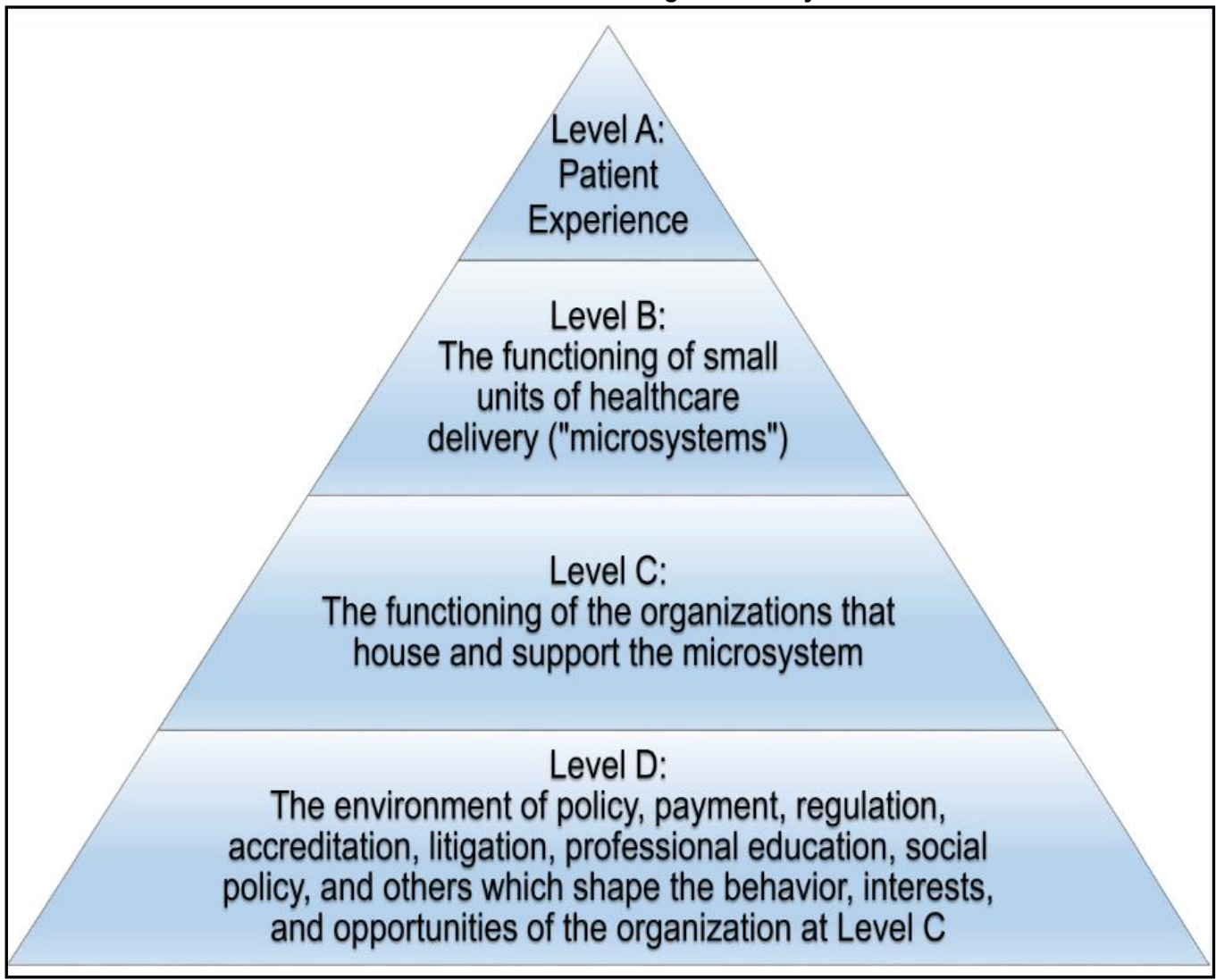

Therefore, analysis of patient harm resulting from error requires not only consideration of an individual provider's actions but also a broader approach with consideration of all system and environmental contributions to error. If contributing system factors are not identified and corrected, the same conditions exist for the same error to occur again with other patients and/or providers. ${ }^{2,4} \mathrm{~A}$ system approach to error analysis recognizes error as the consequence of system factors. ${ }^{4}$ It emphasizes that all factors must be considered before passing judgment on an individual provider. It recognizes that human error only results in an adverse event when multiple layers of safeguards or barriers fail and allow the error to reach the patient. These barriers fail because of a combination of two factors: active failures and latent conditions. ${ }^{4}$ Active failures are actions of providers who are in direct contact with the patient and may include "slips, lapses, fumbles, mistakes, and procedural violations." ${ }^{4}$ While active failures may immediately affect the patient, sometimes the effect is delayed, and sometimes, the patient is never affected. . $^{1,34}$ Latent conditions are organizational factors not in direct contact with the patient. ${ }^{3,4}$ Latent conditions create environments that propagate error (e.g. fatigue, lack of training, productivity expectations, poor communication methods) and can also weaken safeguards against error. ${ }^{4}$ Latent conditions can lie dormant for long periods of time before combining with active failures and allowing adverse events to occur. ${ }^{1,3,4}$

As illustrated in Figure 2, adverse events, as the result of active failures and latent conditions, occur on a continuum of harm ranging from a minor near miss to a major event depending on the severity of harm that occurs. ${ }^{6} \mathrm{~A}$ facility is more likely to investigate a catastrophic event compared to a potentially serious situation that is discovered and corrected before a major event occurs (a major near miss). 6,7 Yet, near misses occur more frequently than actual harmful events and should be investigated as warning signs of future system failure. ${ }^{6}$ 
Figure 2. Continuum of medical error and near miss based on the degree of harm caused by an event; adapted from Ginsburg et al ${ }^{6}$

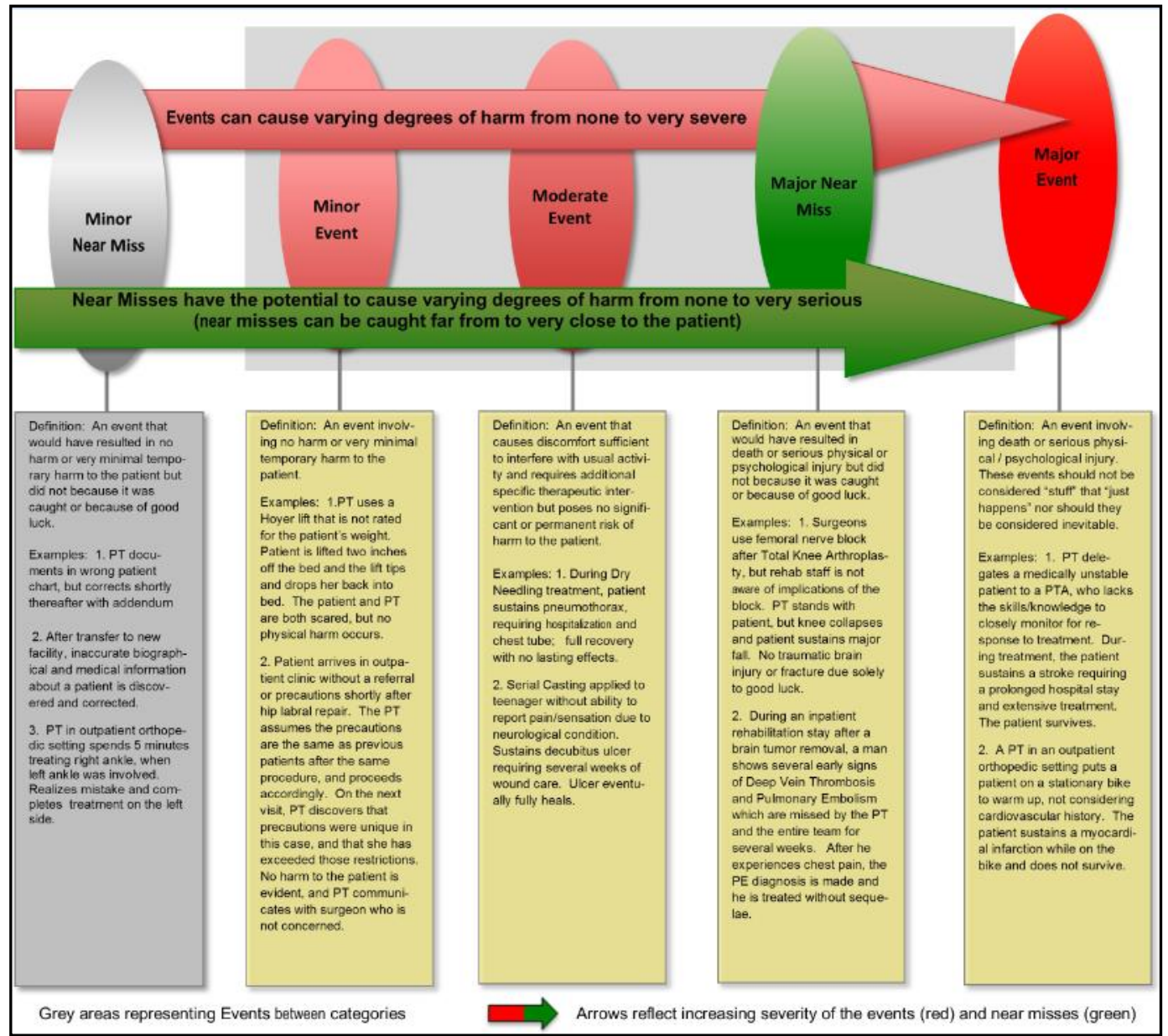

While the incidence of adverse events and methods of error analysis and reduction have been researched in other areas of health care such as medicine, nursing, and pharmacology, few studies address physical therapy error and the analysis of system conditions in rehabilitation settings. ${ }^{8,9}$ Physical therapists may be less involved than other medical providers in error reduction efforts because physical therapy is often perceived as a lower risk profession in that the errors made by therapists may be less likely to be life-threatening. ${ }^{9}$ Therapists may also be hesitant to openly admit error due to the erroneous assumption that reporting will automatically result in punishment or claims of malpractice.8,10,11 However, serious error can and does occur in physical therapy. ${ }^{8}$

PURPOSE: This case report analysis illustrates how both individual and system factors contribute to error in a rehabilitation setting and how identification of these factors allows development of methods to improve future patient safety.

\section{CASE DESCRIPTION}

This case occurred on the brain injury unit of a rehabilitation hospital in the United States. The unit had two interdisciplinary teams, each named for the physiatrist leading the team. The teams were comprised of physicians, physician assistants, nurses, physical therapists, occupational therapists, speech-language pathologists, pharmacists, neuropsychologists, and case managers. Additional coverage staff as well as interns and students of all healthcare disciplines frequently joined and left the team. There was no set combination of providers on each team beyond the physician, so typically no two patients shared the same combination of providers. In this case, physical therapy was provided by a third year Doctor of Physical Therapy intern working under the direct supervision of a physical therapist. 
Patients received therapy five days per week. Their care was divided according to each disciplines' specialty area, and team members met weekly to discuss evaluation results and progress. Each discipline documented weekly in an electronic medical record (EMR) system, and the physician also handwrote notes and orders in a traditional paper chart stored on the unit.

The patient involved was a male in his thirties who was admitted to the hospital two weeks after the resection of a benign meningioma and subsequent right intracranial bleed. The medical record stated that he had no significant past medical history including no personal or family history of a clotting disorder. Vascular ultrasound assessments routinely performed upon admission revealed superficial vein thrombosis (SVT) in his left forearm, but no signs of deep vein thrombosis (DVT). Signs of SVT and DVT were not present in his other three extremities. The patient was not placed on DVT prophylaxis because he was mobile and at a high risk of bleeding due to his recent intracranial bleed. Instead, he received 80 milligrams of aspirin daily. ${ }^{12}$ The physician cleared the patient to participate in therapy with monitoring of his SVT. (See Figure 3 for a timeline of events occurring during the patient's rehabilitation stay.)

Figure 3. Timeline of Events

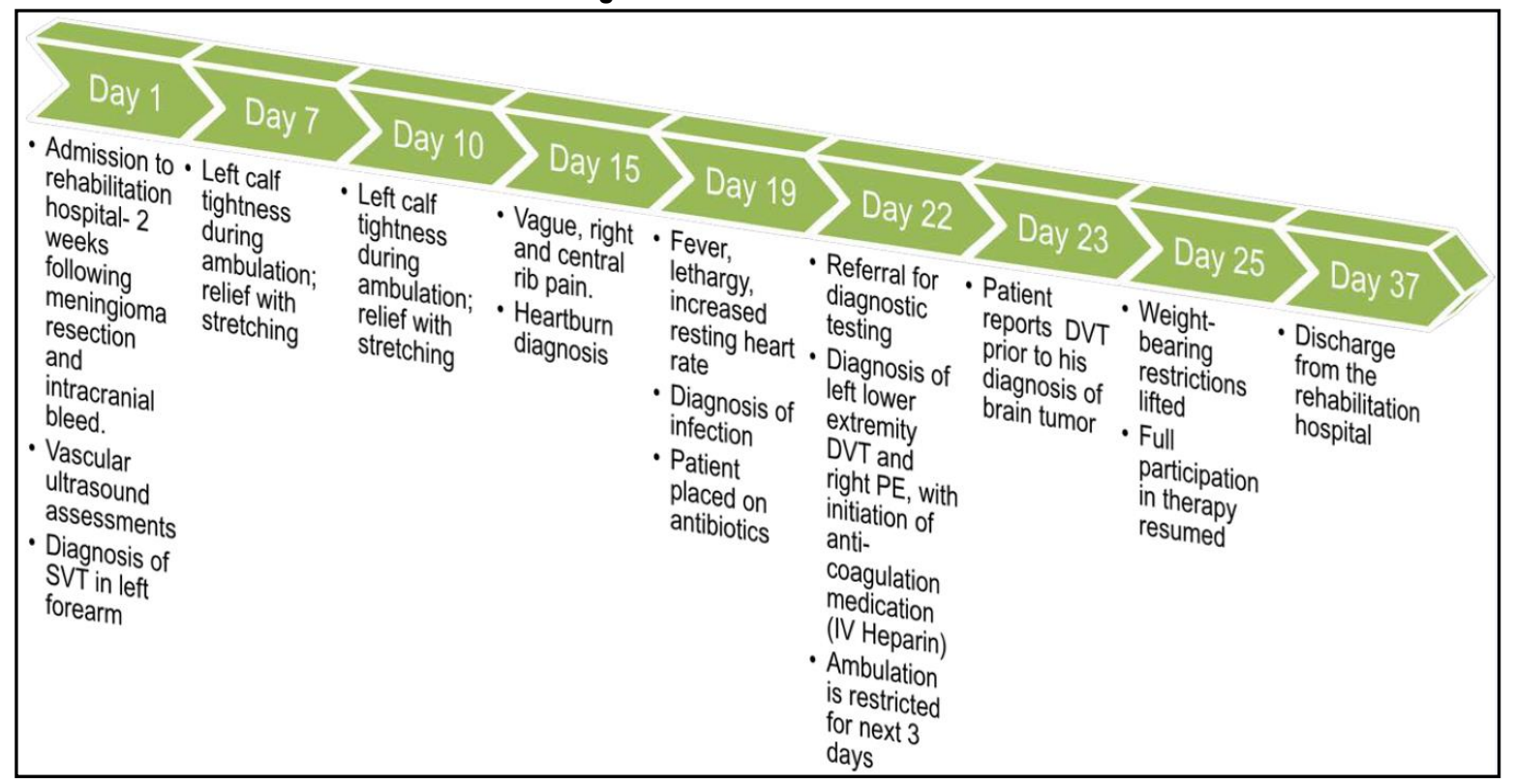

Consistent with the location of his brain injury, the patient presented with left hemiparesis. During ambulation, he required assistance to maintain balance, had ataxia, and demonstrated significant gait deviations that worsened with fatigue. He was alert, oriented, and demonstrated good communication and awareness of his safety and physical deficits. The speech-language pathologist performed cognitive testing and reported that the patient only had mild impairments in processing speed and working memory.

Seven days after admission, the patient reported left calf tightness while ambulating 150 meters. The physical therapist intern considered the possibility of a DVT and determined the patient's risk of clots by weighing the results of a clinical examination, current medical management, and application of the Wells Criteria, a clinical prediction rule widely used to determine if referral for further diagnostic testing is warranted. ${ }^{13}$ Examination revealed no swelling or tenderness in the lower extremity. Examination alone has poor psychometric properties for the detection of DVT, but together with information from the medical history, examination findings help determine a patient's risk. ${ }^{13}$ Recent vascular assessments were negative, and he was on aspirin. ${ }^{12,13}$ Using the Wells Criteria, the intern determined the patient had a moderate risk (17\%) of DVT due to limb paresis and recent surgery. ${ }^{13}$ After discussion with her supervising therapist, the intern decided that an alternate diagnosis of muscle cramping was likely causing the calf tightness because muscle fatigue had already been hypothesized as a contributor to the patient's gait deviations. The patient performed a standing calf stretch which resolved his symptoms. Three days later, left calf tightness returned, and again his symptoms resolved with stretching. The intern documented these events in the physical therapy progress note.

During a mid-morning session five days later, the patient reported vague, right-sided and central chest pain lasting approximately eight minutes while performing standing balance activities. Symptoms were not reproduced with deep breathing or active upper extremity range of motion. The situation was discussed with the supervising therapist and physician assistant who attributed the 
symptoms to heartburn. The intern wondered about this diagnosis because the patient denied a history of reflux and because he had not eaten within the past couple hours. However, neither the physical therapist nor physician assistant seemed concerned, so the intern deferred to their assessment out of respect for their experience and areas of expertise. The intern documented the event in the progress note but was not aware if the physician assistant also documented the event or informed the physician. The patient denied chest pain in subsequent therapy sessions.

Four days later, the patient presented with fever, lethargy, and increased resting heart rate (120 bpm). The physician suspected an infection and prescribed antibiotics. After three days without improvement, the physician referred the patient for further diagnostic testing which revealed left proximal lower extremity DVT and right pulmonary embolism (PE). The patient was given intravenous Heparin and two liters of continuous oxygen. The physician restricted ambulation for the next three days, consistent with the traditional prescription for DVT to prevent the dislodgment of clots. ${ }^{14}$ The intern was curious about this order as current research supports ambulation immediately to early (0-2 days) after a therapeutic level of anticoagulation is reached. ${ }^{14}$ Early ambulation has not been shown to increase the risk of new PE or extension of current DVT, and patients who ambulate early have significantly reduced pain, swelling, and heaviness compared to patients placed on bed rest. ${ }^{14}$ However, the intern did not discuss this order with the physician because she felt she could not immediately support her concerns with current evidence nor did she feel comfortable challenging the experienced physician.

After his PE diagnosis, the patient told the intern and supervising therapist that he had a lower extremity DVT prior to his brain tumor; important historical information because prior thromboembolism increases the risk of a subsequent clot.15,16 The intern documented this missed information but did not verbally inform the team.

Left calf pain, shortness of breath, and decreased cardiovascular endurance were present from PE diagnosis through discharge. Despite ongoing symptoms, rehabilitation goals were met, and he was discharged thirty-seven days after admission. He returned to the same facility for outpatient therapy, and eventually his symptoms resolved.

\section{REFLECTIONS ON THE CASE:}

A three-week delay occurred between the patient's initial symptoms and his DVT/PE diagnosis; a major near miss due to high risk of death from PE. The healthcare team and patient were simply lucky, as an estimated 1 in 5 patients with PE die immediately while an additional $40 \%$ die within 3 months. ${ }^{16}$ Because the patient recovered without serious injury, neither the intern nor interdisciplinary team recognized the event as a significant medical error, and consequently, did not complete an error analysis. The team may have discussed this case with the intent of determining if system factors beyond the innate difficulty of diagnosing these conditions may have contributed to the delayed diagnosis. Identification of these factors could have allowed correction of issues with the goal of preventing future patients from developing PEs. In fact, this case is ideal for learning because direct patient harm was averted, and therefore, the tragedy, guilt, sadness, and risk of litigation are less than if the patient had died. ${ }^{6}$ The same system conditions exist, but they can be examined with fewer emotional barriers.

The intern only recognized error when she retrospectively reflected on the patient's care after completing her clinical experience at the facility. She initially blamed herself, but upon further reflection and review of the literature on medical and system error, she realized other factors might have contributed to the delayed diagnosis and near miss. She completed an analysis to identify contributing factors on each level of the healthcare system (Figure 1). ${ }^{5}$ She utilized a Contributory Factors Checklist to help ensure that factors at all levels of healthcare were considered. 
Table 1. Contributory Factors Checklist. Adapted from the Institute for Healthcare Improvement (IHI) ${ }^{17}$ and from Contributory Factors Framework in Root Cause Analysis-Application Guidelines. ${ }^{18}$

\begin{tabular}{|c|c|c|}
\hline $\begin{array}{l}\text { Patient Factors } \\
\text { Clinical } \\
\text { Pre-existing Co-morbidity } \\
\text { Difficulty in Diagnosis } \\
\text { Personal } \\
\text { Personality } \\
\text { Cultural or Religious beliefs } \\
\text { Social/Family Circumstances } \\
\text { Stress } \\
\text { Disclosure of health history } \\
\text { Interpersonal } \\
\text { Patient-Staff relationship }\end{array}$ & $\begin{array}{l}\text { Staff Factors } \\
\text { Competence } \\
\text { Inadequate Knowledge } \\
\text { Inadequate Skill/Experience } \\
\text { Compliance } \\
\text { Failure to comply with policy } \\
\text { Intentional or unintentional violation } \\
\text { Personal } \\
\text { Personality } \\
\text { Stress } \\
\text { Fatigue } \\
\text { Distraction } \\
\text { Mental Impairment (e.g. illness, drugs, } \\
\text { pain) } \\
\text { Specific illness (e.g. depression) } \\
\text { Domestic Issues } \\
\text { Interpersonal } \\
\text { Staff-patient relationship } \\
\text { Staff-staff/team relationship } \\
\text { Staff-organization relationship }\end{array}$ & $\begin{array}{l}\text { Team Factors } \\
\text { Verbal communication } \\
\text { Between professions } \\
\text { Outside of micro-work environment } \\
\text { Inadequate delegation communication } \\
\text { Written Communication } \\
\text { Incomplete/inadequate information } \\
\text { Discrepancies in notes } \\
\text { Incomplete documentation } \\
\text { Illegible charting } \\
\text { Misinterpretation of information } \\
\text { Supervision and seeking help } \\
\text { Unwillingness to seek help } \\
\text { Unavailability of staff to help } \\
\text { Responsiveness of staff to request for help } \\
\text { Inadequate delegation communication } \\
\text { Congruency } \\
\text { Definition of tasks between professions } \\
\text { Definition of tasks within profession } \\
\text { Leadership } \\
\text { Ineffective leadership } \\
\text { Unclear definitions of responsibility } \\
\text { Staff/colleagues response to incidents } \\
\text { Inadequate support by peers after incident } \\
\text { Inadequate support by team members after } \\
\text { incident }\end{array}$ \\
\hline \multirow{2}{*}{$\begin{array}{l}\text { Work/Care Environment } \\
\text { Building/Design } \\
\text { Inadequate equipment maintenance } \\
\text { Poor functionality (ergonomic } \\
\text { design) } \\
\text { Physical Environment } \\
\text { Movement of patients between } \\
\text { areas } \\
\text { Storage } \\
\text { Inadequate space within rooms } \\
\text { Information Technology } \\
\text { Malfunction/reliability } \\
\text { Functionality of EHR } \\
\text { System Design/Access } \\
\text { Staffing } \\
\text { Unavailability } \\
\text { Allocation of staff } \\
\text { Recruitment } \\
\text { Education/Training } \\
\text { Orientation } \\
\text { Ongoing/refresher training } \\
\text { Workload/Hours of work } \\
\text { Inadequate regular breaks } \\
\text { Heavy workload } \\
\text { Long working hours }\end{array}$} & \multirow{2}{*}{$\begin{array}{l}\text { Management/ } \\
\text { Organizational Factors } \\
\text { Leadership/Governance } \\
\text { Leadership presence } \\
\text { Leadership style } \\
\text { Resources/constraints } \\
\text { Human Resources } \\
\text { Financial Resources } \\
\text { Safety, Culture, Priorities } \\
\text { Inadequate safety culture } \\
\text { Wrong priorities } \\
\text { Organizational Structure } \\
\text { Hierarchical arrangement of staff } \\
\text { Unclear roles/responsibilities } \\
\text { Standardization of common processes } \\
\text { Authority gradient } \\
\text { Objectives, policies/standards } \\
\text { Operations } \\
\text { Human Resources Policies } \\
\text { Information Policies } \\
\text { Risk Management process }\end{array}$} & $\begin{array}{l}\text { Task/Technology Factors } \\
\text { Availability of health information: } \\
\text { Misinterpretation of information } \\
\text { Availability/Reliability of information } \\
\text { Hard to find information in EHR } \\
\text { Task Design } \\
\text { Ease of task execution } \\
\text { Design deficiency }\end{array}$ \\
\hline & & $\begin{array}{l}\text { External Factors } \\
\text { Political } \\
\text { Goals } \\
\text { Perceptions } \\
\text { Economic } \\
\text { Laws/Regulations } \\
\text { Regulatory Requirements with external } \\
\text { Partnership working wip } \\
\text { organizations } \\
\text { Contractual arrangements } \\
\text { Communication }\end{array}$ \\
\hline
\end{tabular}

(C) The Internet Journal of Allied Health Sciences and Practice, 2016 


\section{OUTCOME OF ANALYSIS:}

Multiple system factors were identified as contributing to the delayed diagnosis of the patient's DVT and PE. Key active failures included 1) the interdisciplinary team did not identify a complete past medical history, 2) the intern, physician assistant, and supervising physical therapist did not directly report the patient's symptoms to the physician, leading the physician to make clinical decisions without key information, 3) the intern did not seek clarification from the physician and physician assistant about their clinical decisions, and 4) the intern did not use best interventions supported by research. Latent system conditions that contributed to and combined with each of these active failures are summarized below:

Several latent conditions were identified as contributors to the team's failure to learn about the patient's history of DVT (See subcategories of Table 1) $)^{17,18}$ This team typically worked with patients who had decreased ability to communicate, which combined with the trauma and complexity of the situation, made accurate identification and verification of information difficult. Therefore, the team relied on the accuracy of the historical record of past treating institutions (Task/Technology Factors, External Factors). The team did not recognize and utilize this patient's "unique" ability to verify his medical history (Patient Factors). Finally, beyond performing ultrasound assessments, the facility did not utilize a standardized set of questions or process to screen for DVT or other common complications (Management/Organizational Factors).

The cause of the patient's calf tightness cannot be definitely known, but in retrospect, the intern should have directly informed the physician because the patient's medical condition, including his current SVT, automatically increased his risk of DVT. 15,16,19 However, latent conditions including the weekly occurrence of rounds (Team Factors), high reliance on verbal communication (Team Factors), challenging EMR design (Task/Technology Factors), and poor daily communication methods (Team Factors) also contributed to the physician not knowing about the symptoms. No formal methods of daily communication were available beyond reporting in weekly rounds or physically locating the physician for a conversation. By the time rounds occurred several days after the patient's symptoms resolved, the intern had other, more current information to report. Because each disciplines' notes were written weekly and were not easily accessible to other members of the team, the physician likely never saw the documentation of calf tightness or chest pain. The physician may not have known about the symptoms experienced by the patient because of limited bi-directional communication amongst team members. Effective communication between team members requires a balance of both verbal and written communication. Alone, verbal communication is prone to information loss due to interruptions, fatigue, or boredom, and typically, verbal interaction occurs between a few people while the rest of the team remains uninformed.9,20,21 Similarly, solely relying on written documentation can also lead to missed information as EMR systems often contain large amounts of information, making it difficult to find the most critical and pertinent information during a chart review.

Latent conditions also contributed to the intern's failure to seek clarification about the medical team's decisions which then led to the failure to use the best interventions following thrombotic complications. The physician was designated as the leader of the team by the facility, and therefore, was automatically placed in a position of authority (Management/Organizational Factors). Additionally, the team consisted of professionals who were highly skilled in their areas of specialization. These factors created perceived authority gradients between disciplines. An authority gradient is defined as "the balance of decision-making power or the steepness of command hierarchy in a given situation."22 As a result of the authority gradients, the intern, and likely other team members, did not challenge or seek clarification from the leader or other colleagues because of feeling that the experience and knowledge of other providers should be respected. ${ }^{22,23}$ These gradients can also create an overreliance on other team members to monitor and treat a particular body system.

Finally, an important latent condition was the presence of the intern under the supervision of a licensed physical therapist (Staff Factor). Due to limited clinical experience, the intern did not yet have a strong professional identity and was at times uncertain if her level of concern was appropriate. The intern's automatic first step in any questionable situation was to discuss the issue with her supervisor. On a positive side, this discussion creates another protective layer against error. However, interns may actually have more information about recent literature, but because of perceived inferiority of knowledge and experience, may not act on what they think is best and instead use their supervisors' responses to decide if their concern is justified.22

The analysis also identified what system factors worked well. In other words, what "defenses" or "safeguards" stopped the sequence of events from resulting in permanent harm or death. ${ }^{4}$ While luck was involved, other factors such as the facilities' ability to quickly and efficiently coordinate medical imaging with a neighboring facility (External Factor), the physician's experience and response with correct medication management once the DVT and PE were identified (Staff Factor), and the physician's efficient communication with the nursing staff and pharmacist to ensure appropriate adminstration of anticoagulation medications (Team Factor) all contributed to a successful outcome.

(C) The Internet Journal of Allied Health Sciences and Practice, 2016 


\section{DISCUSSION}

An analysis of this case led to identification of both individual and system factors that contributed to this major near miss, demonstrating that physical therapists and other allied health professionals can and should consider how systemic factors contribute to their own clinical actions and decisions. Professional autonomy brings with it the responsibility to consider all risk factors and to advocate for patient safety. ${ }^{8}$ When individuals and facilities acknowledge that error is inevitable and not isolated to the actions of one person, a shift can begin to occur from blaming individuals to reporting, analyzing, and learning from mistakes. ${ }^{8,10}$

The issues identified in this case analysis are not unique to this intern or facility. A qualitative study identified common errors made by physical therapists (3-39 years of practice) across multiple clinical settings. ${ }^{8}$ The common active failures included failure of the therapist to communicate in a confident manner and to share information with other providers; failure to demonstrate professional autonomy and acknowledge responsibility for physical therapy issues; and implementation of physician's orders when evidence supports other more effective interventions. ${ }^{8}$ The qualitative study, along with other studies in the medical literature, identified latent conditions including barriers to accessing medical records; lack of methods that support timely and effective communication; the presence of authority gradients; and lack of skills needed to initiate conversations about patient safety. 1,8,9,20,23-25 Additional factors related to healthcare interns included concerns over being negatively evaluated, difficulty finding appropriate words to express concern, high regard for the instructor-intern relationship, and respect for the instructor's experience. ${ }^{23,24}$

\section{Prevention of Future Error}

Methods of remediating the specific active failures and latent conditions in this case are cited in the medical literature and may be applied in the case. $1,5,8,9,20,21,24-30$ To our knowledge, no studies published within the physical therapy literature investigate the effectiveness of these interventions at reducing error.

\section{a. Changes to Team Dynamics and Communication (Level B)}

To ensure effective collaboration, medical teams should be trained to work together. ${ }^{1}$ Formal teamwork training programs, such as TeamSTEPPS, teach groups to develop an attitude of "looking out for each other."1,11,26,27 This training educates team members about their roles and encourages disciplines to proactively cross-check each other's work, seeking solutions to patient safety issues together without compromising the strong knowledge base of individual disciplines. ${ }^{1,26}$ Ideally, all members join the team and are trained at the same time in order to maximize collaboration and develop common goals and vision. ${ }^{27}$ If other individuals intermittently join the team, active processes must be in place to ensure that new staff understands their responsibilities and current team procedures. Institutions should also consider how frequent team variability potentially weakens effective teamwork and creates system vulnerability. ${ }^{27}$

Leaders can reduce authority gradients by encouraging members to voice their opinions and by sharing past experiences in which they made poor clinical decisions. $1,5,24$ These admissions help new, inexperienced team members understand that everyone is vulnerable to error. 8,24 Facilities and teams should consider if the same person (in this case the physician) should always be designated as the leader or if this structure inherently creates an authority gradient. Rather, should circumstances and patientneed dictate the leader of the team?

Methods to improve communication and lower the rate of adverse events include brief daily rounds (sometimes called "lightning rounds") to discuss new developments and immediate concerns while saving in-depth discussions for weekly planning meetings; structured communication protocols for daily rounds (e.g. SBAR); and development of an EMR system with easier access to documentation and pop-up notifications to support verbal communication and alert the entire team of new issues.20,21,27-29

The importance of the patient and family in error reduction is often underappreciated..$^{1,930}$ If the situation is appropriate, patients and caregivers should be educated about the symptoms of potential complications and empowered to speak up if they are concerned. 30

\section{b. Changes to Facility Operations (Level C)}

Standardized protocols and checklists are used in aviation to ensure safety of passengers, while surgical teams utilize protocols no matter how many times they have performed a procedure in order to ensure safety of patients. $3,9,31$ Interdisciplinary teams may similarly benefit from use of protocols for the screening, identification, and management of high risk issues and complications that frequently occur in their patient population. When all team members are responsible for following a protocol, the risk of error is greatly reduced and blame is removed from one team member. ${ }^{3,31}$ 


\section{c. Changes to Entry-Level and Continuing Physical Therapy Education (Level D)}

The concepts of active failure and latent conditions should be formally included in physical therapist education. ${ }^{23,25} \mathrm{~A}$ study examining the attitudes and behaviors of fourth year medical students suggests that without formal education, the majority of students lack the confidence and skills to report errors appropriately and to seek clarification from their instructors. ${ }^{25}$ Within physical therapist education, interns and students need opportunities to practice formal communication methods in both clinical and simulated environments. ${ }^{23-25}$ Faculty and clinical educators also need to be educated about responding appropriately to inquiries from their students, modeling correct behaviors, and demonstrating that despite their position of seniority, they still value and respect the contributions of their students. ${ }^{25}$

It is important to include experienced clinicians in education and training about error reduction processes. The skill of recognizing, reporting, and preventing error can be developed through simulations and case discussions. Purposeful analysis of actual error has a greater impact for learning than general discussion of error. With practice, therapists gain the confidence to demonstrate professional autonomy and help change system contributions to error. ${ }^{8}$

\section{CONCLUSION:}

In this rehabilitation case example, individual mistakes and system factors on several levels of the healthcare system were identified as contributors to the adverse event. The medical literature and the few physical therapy papers on this topic propose similar common contributors to error, suggesting that these factors are not unique to this case. Physical therapists, as members of wider healthcare teams, are obligated to improve patient safety by applying current knowledge of common errors and developing formal error reduction processes within their practice settings.

\section{References}

1. Kohn LT, Corrigan J, Donaldson MS, eds. To Err is Human: Building a Safer Health System. Washington, D.C.: National Academy Press; 2000.

2. Reason JT, Carthey J, de Leval MR. Diagnosing "vulnerable system syndrome": an essential prerequisite to effective risk management. Qual Health Care. 2001;10 Suppl 2:ii21-5. [PMID: 11700375]

3. Reason J. Human Error. Cambridge: Cambridge University Press; 1990.

4. Reason J. Human error: Models and management. West J Med. 2000;172(6):393-6. [PMID: 10854390]

5. Berwick DM. A user's manual for the IOM's 'Quality Chasm' report. Health Aff (Millwood). 2002;21(3):80-90. [PMID: 12026006]

6. Ginsburg LR, Chuang YT, Richardson J, et al. Categorizing errors and adverse events for learning: a provider perspective. Healthc Q. 2009;12:154-60. [PMID: 19667794]

7. Greenberg CC. Learning from adverse events and near misses. J Gastrointest Surg. 2009;13(1):3-5. [PMID: 18797974]

8. Cochran TM, Mu K, Lohman H, Scheirton LS. Physical therapists' perspectives on practice errors in geriatric, neurologic, or orthopedic clinical settings. Physiother Theory Pract. 2009;25(1):1-13. [PMID: 19140077]

9. King J, Anderson CM. Patient safety and physiotherapy: what does it mean for your clinical practice? Physiother Can. 2010;62(3):172-9. [PMID: 21629594]

10. Anderson JC, Towell ER. Perspectives on assessment of physical therapy error in the new millennium. Journal of Phys Ther Educ. 2002;16(3).

11. Galt KA, Paschal KA. Foundations in Patient Safety for Health Professionals. Sudlbury, MA: Jones \& Bartlett Learning; 2009.

12. Serebruany VL, Steinhubl SR, Berger PB, et al. Analysis of risk of bleeding complications after different doses of aspirin in 192,036 patients enrolled in 31 randomized controlled trials. Am J Cardiol. 2005;95(10):1218-22. [PMID: 15877994]

13. Riddle DL, Wells PS. Diagnosis of lower-extremity deep vein thrombosis in outpatients. Phys Ther. 2004;84(8):729-35. [PMID: 15283623]

14. Aissaoui N, Martins E, Mouly S, Weber S, Meune C. A meta-analysis of bed rest versus early ambulation in the management of pulmonary embolism, deep vein thrombosis, or both. Int J Cardiol. 2009;137(1):37-41. [PMID: 18691773]

15. Anderson FA Jr, Spencer FA. Risk factors for venous thromboembolism. Circulation. 2003;107(23 Suppl 1):19-16. [PMID: 12814980]

16. Office of the Surgeon General (US); National Heart, Lung, and Blood Institute (US). The Surgeon General's Call to Action to Prevent Deep Vein Thrombosis and Pulmonary Embolism. Rockville (MD): Office of the Surgeon General (US); 2008: http://www.ncbi.nlm.nih.gov/books/NBK44178/.

17. Institute for Healthcare Improvement: Open School. PS 104: Root Cause and System Analysis. 2014; http://www.ihi.org/education//HIOpenSchool/Courses/Documents/Course\%20Summaries.pdf. Accessed November 2, 2014.

18. Emslie S. Root Cause Analysis-Application Guidelines. 2007; https://stuart4handouts.files.wordpress.com/2011/04/moh-rcaguidelines-july-2007_b.pdf. Accessed November 4, 2015.

19. Marchiori A, Mosena L, Prandoni P. Superficial vein thrombosis: risk factors, diagnosis, and treatment. Semin Thromb Hemost. 2006;32(7):737-43. [PMID: 17024602]

(c) The Internet Journal of Allied Health Sciences and Practice, 2016 
20. Collins SA, Bakken S, Vawdrey DK, Coiera E, Currie L. Model development for EHR interdisciplinary information exchange of ICU common goals. Int J Med Inform. 2011;80(8):e141-9. [PMID: 20974549]

21. Edwards C. Using interdisciplinary shared governance and patient rounds to increase patient safety. Medsurg Nurs. 2008;17(4):255-7. [PMID: 18807861]

22. Agency for Healthcare Research and Quality (AHRQ): Glossary: Authority Gradient. 2014; http://psnet.ahrq.gov/popup_glossary.aspx?name=authoritygradient. Accessed December 9, 2015.

23. Pian-Smith MC, Simon R, Minehart RD, et al. Teaching residents the two-challenge rule: a simulation-based approach to improve education and patient safety. Sim Healthcare. 2009;4(2):84-91. [PMID: 19444045]

24. Cosby KS, Croskerry P. Profiles in patient safety: authority gradients in medical error. Acad Emerg Med. 2004;11(12):13415. [PMID: 15576526]

25. Wetzel AP, Dow AW, Mazmanian PE. Patient safety attitudes and behaviors of graduating medical students. Eval Health Prof. 2012;35(2):221-38. [PMID: 21788294]

26. Agency for Healthcare Research and Quality (AHRQ), Patient Safety Network, Patient Safety Primer: Teamwork Training. 2013; http://psnet.ahrq.gov. Accessed November 20, 2015.

27. Bharwani AM, Harris GC, Southwick FS. Perspective: a business school view of medical interprofessional rounds: transforming rounding groups into rounding teams. Acad Med. 2012;87(12):1768-71. [PMID: 23095926]

28. O'Leary KJ, Buck R, Fligiel HM, et al. Structured interdisciplinary rounds in a medical teaching unit: improving patient safety. Arch Intern Med. 2011;171(7):678-84. [PMID: 21482844]

29. Vazirani S, Hays RD, Shapiro MF, Cowan M. Effect of a multidisciplinary intervention on communication and collaboration among physicians and nurses. Am J Crit Care. 2005 Jan;14(1):71-7. [PMID: 15608112]

30. Vincent CA. Patient safety: what about the patient? Qual Saf Health Care. 2002;11(1):76-80. [PMID: 12078376]

31. Agency for Healthcare Research and Quality (AHRQ): Patient Safety Network, Patient Safety Primer: Checklists. 2013; http://psnet.ahrq.gov. Accessed November 30, 2015. 